\title{
EXACT COLIMITS
}

\author{
BY JOHN ISBELL AND BARRY MITCHELL
}

Communicated by Hyman Bass, March 29, 1973

It is well known and easy that if $\boldsymbol{C}$ is a small category with filtered components, then the functor colim ${ }_{C}: \mathrm{Ab}^{c} \rightarrow \mathrm{Ab}$ is exact. The converse was conjectured and proved in a special case by Oberst [4]. A necessary and sufficient condition for exactness of colim $_{c}$ was given by Isbell in [2], who used the condition to show that Oberst's conjecture is true when $\boldsymbol{C}$ is a monoid. We show that the conjecture is false in general. Proofs will only be sketched here, full details to appear elsewhere.

1. Affinization. If $A$ and $B$ are objects of $C$, then $A$ maps to $B$ if $C(A, B)$ is nonempty. If $\alpha_{i}$ is a family of $C(A, B)$, then $\beta$ filters the family if $\beta \alpha_{i}$ is independent of $i$. A category $C$ is filtered if every pair (and hence every finite family) of objects map to a common object, and every pair (and hence every finite family) of morphisms with common domain and codomain are filtered.

The additivization of $\boldsymbol{C}$ is the category $\boldsymbol{Z C}$ with the same objects, where $Z C(A, B)$ is the free abelian group on $C(A, B)$. The affinization of $C$ is the subcategory of $\boldsymbol{Z C}$ of morphisms whose integer coefficients sum to one. Note that $\boldsymbol{C} \subset$ aff $\boldsymbol{C}$, with equality if and only if $\boldsymbol{C}$ is a preordered set.

If $M \in \mathrm{Ab}^{C}$, then $\operatorname{colim}_{C} M=\oplus_{A \in|C|} M(A) / X$ where $X$ is the subgroup of the numerator generated by elements of the form $x-\alpha x$ with, say, $x \in M(A), \alpha \in C(A, B)$, and hence $\alpha x \in M(B)$. Note that if $\sum n_{i} \alpha_{i}$ is a morphism of aff $C$, then

$$
x-\left(\sum n_{i} \alpha_{i}\right) x=\sum n_{i}\left(x-\alpha_{i} x\right),
$$

and it follows that if $M$ is considered as an object of $A b^{\text {aff } c}$ in the obvious way, then $\operatorname{colim}_{c} M=\operatorname{colim}_{\text {aff } c} M$. This yields easily the "if' part of the following theorem, which is close to being a restatement of [2, Theorem 1$]$.

THEOREM 1. Colim $c$ is exact if and only if the components of aff $C$ are filtered.

The converse is an application of the "several object" version of ring theory [3]. We express the colimit $\cdots$ as colim $M=\Delta Z \otimes_{z C} M$ where $\Delta \boldsymbol{Z}$ is the constant functor at $\boldsymbol{Z}$ over $\boldsymbol{C}^{\text {op }}$. Then exactness of $\operatorname{colim}_{\boldsymbol{C}}$ is

AMS (MOS) subject classifications (1970). Primary 18G99; Secondary 18E05.

Key words and phrases. Affinization, diagram, colimit. 
equivalent to flatness of $\Delta \boldsymbol{Z}$, which in turn is equivalent to purity of

$$
0 \rightarrow K \rightarrow \underset{B \in|C|}{\bigoplus} Z C(\quad, B) \stackrel{\varepsilon}{\longrightarrow} \Delta Z \rightarrow 0
$$

where $\varepsilon$ sums coefficients. Using P. M. Cohn's equational characterization of purity and the following obvious lemma, the other part of Theorem 1 is only a couple of easy steps away.

Lemma 1. Pairs of morphisms in aff $\boldsymbol{C}$ can be filtered in aff $\boldsymbol{C}$ if and only if finite families of morphisms in $\boldsymbol{C}$ can be filtered in aff $\boldsymbol{C}$.

THEOREM 2. If colim $_{c}$ is exact, then any pair $\alpha, \alpha e$ with e an endomorphism can be filtered.

One need only observe that the proof given in [2] for $\boldsymbol{C}$ a monoid never uses the fact that $\alpha$ is an endomorphism. The proof can also be reduced from two cases to one using the neater formulation of Theorem 1.

A weak terminal object of a category is an object to which all objects map.

COROLlaRy 1. If colim $_{C}$ is exact, and if all components of $C$ have weak terminal objects, then the components of $\boldsymbol{C}$ are filtered.

This has also been observed by W. Spears [5].

A one way category (called a delta in [3]) is a category whose only endomorphisms are identities. Every category $\boldsymbol{C}$ has a one way reflection $\hat{\boldsymbol{C}}$, namely, the quotient category obtained by identifying all endomorphisms to identities. If colim ${ }_{C}$ is exact, then colim $_{C}$ is exact (true, more generally, for any quotient category).

COROLlary 2. If colim $_{C}$ is exact, and if two morphisms of $C$ can be filtered in $\hat{\boldsymbol{C}}$, then they can be filtered in $\boldsymbol{C}$.

2. The counterexample. It follows from Corollary 2 that if there is a counterexample to Oberst's conjecture, then there is a one way counterexample. Furthermore it cannot have a weak terminal object by Corollary 1 , and it cannot be a preordered set by Theorem 1 . A candidate arising in nature is the category $\Delta_{\text {face }}$ (which we shall denote simply by $\Delta$ ) of order preserving injections of finite totally ordered sets. This category is as far from being filtered as possible: if two morphisms are filtered, then they are equal.

\section{THEOREM 3. aff $\Delta$ is filtered.}

Let $[n]$ denote $\{0,1, \ldots, n\}$ with the natural order. Let $\Pi(n)$ be the $2^{n+1}-1$ element set of all sequences (including the empty one) of at most $n$ plus and minus signs. $\Pi(n)$ is totally ordered by extending all 
sequences to length $n$ with 0 's, ordering lexicographically with the convention $-<0<+$, and then deleting the 0 's. $\Pi(n)$ also has an obvious partial order, namely, one sequence is greater than another if it extends it. If $\Sigma$ is any subset of $\Pi(n)$, an error for $\Sigma$ is an element $\sigma \in \Pi(n)$ such that $\Sigma$ meets the set $E(\sigma)$ of (not necessarily proper) extensions of $\sigma$, but does not meet every maximal chain in $E(\sigma)$. The set $\Sigma$ is correct if it has no error. A hole of a correct set $\Sigma$ is a sequence $\sigma$ such that $\Sigma$ meets $E(\sigma)$ but $\sigma \notin \Sigma$.

For each $n+1$ element set $\Sigma \subset \Pi(n)$, there is exactly one morphism $q_{\Sigma}:[n] \rightarrow \Pi(n)$ in $\Delta$ with image $\Sigma$. Set $\rho^{n}=\sum(-1)^{h} q_{\Sigma}$ summed over all $n+1$ element correct subsets $\Sigma, h$ being the number of holes of $\Sigma$. One can verify that for each of the $n+1$ morphisms $f:[n-1] \rightarrow[n]$ of $\Delta$, we have $\rho^{n} f=i \rho^{n-1}$ where $i$ is the inclusion $\Pi(n-1) \subset \Pi(n)$. It follows by induction that $\rho^{n}$ is affine. Furthermore, if $f:[n-k] \rightarrow[n]$, then since $f$ factors through all objects between $[n-k]$ and $[n]$, we obtain $\rho^{n} f=i \rho^{n-k}$ where $i$ is now the inclusion $\Pi(n-k) \subset \Pi(n)$. The theorem then follows from Lemma 1.

\section{REFERENCES}

1. N. Bourbaki, Eléments de mathématique. Fasc. XXVII. Algèbre commutative, Actualités Sci. Indust., no. 1290, 1293, Hermann, Paris, 1961. MR 36 \# 146; 30 \#2027.

2. J. R. Isbell, A note on exact colimits, Canad. Math. Bull. 11 (1968), 569-572. MR 39 \#286.

3. B. Mitchell, Rings with several objects, Advances in Math. 8 (1972), 1-161. MR $45 \# 3524$.

4. U. Oberst, Homology of categories and exactness of direct limits, Math. Z. 107 (1968), 87-115. MR 39 \# 5662.

5. W. T. Spears, When is colim exact? (to appear).

Department of Mathematics, State University of New York at Buffalo, Amherst, New YoRK 14226

Department of Mathematics, Rutgers University, New Brunswick, New Jersey 08903

Current address (John Isbell): Instituto di Matematica, Università di Perugia, Perugia, Italia 